\title{
HSPB7 Protected the Osteogenic Differentiation of Mesenchymal Stem Cells From TNF-a Through Chaperone-mediated Autophagy/beta-catenin Pathway
}

\section{Kai Hang}

Zhejiang University School of Medicine Second Affiliated Hospital

\section{Li Ying}

Zhejiang University School of Medicine Second Affiliated Hospital Jinwu Bai

Zhejiang University School of Medicine Second Affiliated Hospital Yibo Wang

Zhejiang University School of Medicine Second Affiliated Hospital

\section{Zhihui Kuang}

Zhejiang University School of Medicine Second Affiliated Hospital Jianx Xu

Zhejiang University School of Medicine Second Affiliated Hospital

Chenw Zhou

Zhejiang University School of Medicine Second Affiliated Hospital

\section{Deting Xue}

Zhejiang University School of Medicine Second Affiliated Hospital

\section{Zhijun Pan ( $\nabla$ zrpzj@zju.edu.cn )}

Zhejiang University School of Medicine Second Affiliated Hospital https://orcid.org/0000-0002-46185990

\section{Research}

Keywords: Heat shock protein B7, TNF-a, osteogenesis, CMA, fracture healing

Posted Date: August 13th, 2021

DOl: https://doi.org/10.21203/rs.3.rs-798091/v1

License: (c) (1) This work is licensed under a Creative Commons Attribution 4.0 International License. Read Full License 


\section{Abstract}

\section{Background}

Managing healing of impaired bone fracture has long been a subject of concern. Prolonged or uncontrolled inflammation exerts detrimental effects on bone healing. Tumor necrosis factor (TNF)- $a$ is a critical inflammatory factor, whose absence impairs normal bone formation. However, TNF-a exposure for longer duration inhibits bone regeneration. In this study, we aim to find a new therapeutic target for the impaired osteogenesis induced by long exposure of TNF-a.

\section{Methods}

In vitro, mRNA microarray analysis was used to identify differentially expressed genes. Cell proliferation assay was used to assess the proliferation of cells. qPCR and Western blotting analysis were applied to detect the expression of target genes and proteins respectively. ALP staining and Alizarin Red staining (ARS) were used to evaluate ALP activity and mineral deposition respectively. Co-immunoprecipitation was used to detect the interaction of proteins. In vivo, a murie tibial fracture model was established, histological evaluation and radiographic analysis was used to confirm bone regeneration in fracture healing. statistical significance between two groups was determined by Student's $t$ test, one-way ANOVA or Bonferroni's post-hoc test according to the distribution of the tested population.

\section{Results}

In this study, we show that heat shock protein family B (small) member 7 (HSPB7) mitigates negative impact of long-term TNF-a exposure on bone formation by binding heat shock protein family $\mathrm{H}$ (small) member 1 (HSPH1). HSPH1, in turn, inhibits the ATPase of heat shock cognate A8 (HSCA8), one of the key components involved in chaperone-mediated autophagy (CMA). Deletion of genes encoding for either HSCA8 or lysosome-associated membrane protein 2A (LAMP2A), another component of CMA, failed to reverse the osteogenic differentiation of hBMSCs induced by TNF-a by deleting HSPH1. Moreover, LAMP2A overexpression reverse the impaired osteogenesis induced by TNF-a, and this effect was attenuated by DKK1, a specific Wnt/ $\beta$-catenin signaling pathway inhibitors. Thus, a heat shock protein family network composed by HAPB7, HSPH1 and HSCA8 rescued the impairment of bone healing by TNF-a through the $C M A / \beta$-catenin pathway, making it a potential therapeutic agent for bone regeneration in cases of prolonged or severe inflammation in the clinical settings.

\section{Conclusions}

Taken together, these findings indicate that a heat shock protein family network including HSPB7, HSPH1 and HSCA8 protects the impaired osteogenesis induced by TNF- $\alpha$ via the CMA/ $\beta$-catenin pathway. And in vivo, HSPB7 overexpression lentiviral particles effectively protects the impaired fracture healing induced by TNF- $a$ in a mouse tibia fracture healing model. 


\section{Introduction}

In the United States, around 8 million people fracture a bone each year, around $5 \%-20 \%$ of these result in impaired or delayed healing, thereby requiring therapeutic intervention eventually, and causing an important clinical and economic issue(Kovach et al., 2015).

An inflammatory response is necessary for the bone regeneration process after injury. A large number of proinflammatory and chemotactic cytokines including tumor necrose factor (TNF)-a, interleukin (IL)-1 $\beta$, IL- 6 and C-C motif chemokine ligand (CCL)2 are released in the early inflammatory phase. Evidence has shown that these mediators not only initiate the repair process by stimulating angiogenesis, but also attract mesenchymal stem cells (MSCs) to the repair region and promote their osteogenic differentiation. Human bone marrow mesenchymal stem cells (BMSCs), also known as multipotent mesenchymal stromal cells, have self-renewal and multipotent differentiation abilities(Hang et al., 2019). Treatment with anti-inflammatory drugs in the early stage of fracture healing markedly impairs the process (Ai-Aql et al., 2008; Claes et al., 2012; Cottrell and O'Connor, 2010; Gerstenfeld et al., 2003b; Loi et al., 2016). However, inflammation is a double-edged sword. Prolonged or uncontrolled inflammation has been shown to have destructive effects on bone healing(Takayanagi, 2009). some chronic inflammation conditions, such as bacterial infection and system immunity, cause impaired fracture healing, which is mainly associated with inflammatory mediators, especially TNF-a(Al-Sebaei et al., 2014; Alblowi et al., 2009; Kayal et al., 2010). To date, there are no effective biotherapy methods to manage harmful inflammatory responses to prevent fracture nonunion.

TNF- $\alpha$ is a critical inflammatory factor in bone fracture healing. On one hand, in the acute inflammatory response phase $24 \mathrm{~h}$ after trauma, TNF-a concentration peaks, and is maintained above baseline for $72 \mathrm{~h}$. TNF-a has been shown to recruit necessary cells such as MSCs for fracture healing, and have shown a pivotal role in fracture healing using TNF-a receptor-deficient mice(Cho et al., 2006; Gerstenfeld et al., 2003a; Gerstenfeld et al., 2003b; Kon et al., 2001; Marsell and Einhorn, 2011). In vitro studies using different doses or exposure times of TNF-a treatment resulted in, contradictory effects of TNF-a on MSC osteogenic differentiation(Ding et al., 2009; Hess et al., 2009; Huang et al., 2011; Lacey et al., 2009; Lu et al., 2012).

Small heat shock proteins (sHSPs) are a family of 12-43 kDa molecular chaperons that are ubiquitously expressed in organisms from prokaryotes to eukaryotes and even in viruses (Maaroufi and Tanguay, 2013). sHSPs consist of 11 members which are characterized by an a-crystallin domain (CAD) flanked by a conserved $\mathrm{N}$-terminal arm and a variable C-terminal extension domain(Maaroufi and Tanguay, 2013). They present varied expression patterns and heterogeneous action mechanisms. While some members of the family are expressed ubiquitously like HSPB1, others are limited to certain areas or tissues, such as heat shock protein family B (small) member 7 (HSPB7)(Kappé et al., 2003; Ke et al., 2011). The sHSPs characteristically bind denaturing proteins to prevent their aggregation and assist in subsequent refolding in collaboration with ATP-dependent chaperons, or to degrade unfolded proteins via proteasomal and/or autophagic pathways (Liao et al., 2017; Mymrikov et al., 2011; Sun and MacRae, 2005; Wu et al., 2017). In 
osteoblasts, stress stimulation induced the expression of HSPB1 which regulated the differentiation of osteoblasts (Shakoori et al., 1992). Among these sHSPs, HSPB7 is one of the least characterized and is highly expressed in the heart, HSPB7 gene mutation is strongly associated with heart diseases (Krief et al., 1999; Mercer et al., 2018). Within the HSPB family of molecular chaperons, HSPB7 is the most potent inhibitor of polyQ protein aggregation (Vos et al., 2010).

There are several types of autophagic pathways in mammals, namely macroautophagy, microautophagy and chaperone-mediated autophagy (CMA) (Kaushik and Cuervo, 2018). In contrast to the other two autophagy pathways, CMA only degrades proteins that contain a specific KFERQ-like motif in their amino acid sequences (Dice, 1990). Chaperoe HSCA8 and co-chaperones recognize KFERQ-like motif proteins, which are then delivered to lysosomes by interacting with the receptor lysosome-associated membrane protein type 2A (LAMP2A), the substrate is then degraded in the lysosomal lumen (Cuervo and Wong, 2014).

In this study, we provide the first evidence that a heat shock protein family composed by HSPB7, HSPH1 and HSCA8 may be a potential therapeutic target for impaired fracture healing in osteomyelitis patients induced by excess of TNF-a through heat shock protein family network.

\section{Results}

\section{TNF-a different treatment times have opposing effects on BMSC osteogenesis}

We performed a Cell Counting Kit (CCK)-8 assay to determine the influence of TNF-a at different concentrations $(0-100 \mathrm{ng} / \mathrm{mL})$ on the proliferation of BMSCs during osteogenic differentiation, we found no significant differences on cell number between different TNF-a concentration groups in the first 6 days (Fig. 2a)

To determine the effect of TNF-a on the osteogenesis of BMSCs, BMSCs were treated with different concentrations of TNF-a during osteogenic differentiation. In the early days (1-2d), TNF-a (1-100 ng/mL) promoted the expression of osteo-specific proteins such as collagen type 1 alpha 1 (Col1A1), runt-related transcription factor 2 (Runx2) and SP7/Osterix (SP7/Osx). However, these osteo-specific proteins were downregulated when BMSCs were treated with TNF-a (1-100 ng/mL) more than $3 \mathrm{~d}(4-5 \mathrm{~d})$ during osteogenesis differentiation (Supplementary Fig. 1).

Interestingly, TNF-a (1-100 ng/mL) enhanced calcium mineralization and alkaline phosphatase (ALP) activity significantly, regardless of treatment duration (Supplementary Fig. 2a)

\section{TNF- $a$ treatment results in $\mathbf{8 9 0}$ differentially expressed genes}


Based on these observations, in order to explore potential targets related to the impairment caused by TNF-a $(100 \mathrm{ng} / \mathrm{mL})$ treatment, we performed an mRNA microarray to determine the comparative transcriptome analysis of 4d TNF-a (100ng/mL)-treated (Os4_T) and control (Os4_C) osteogenic BMSCs. Triplicates of Os4_T and Os4_C samples were collected and subjected to mRNA microarray analysis to identify differentially expressed genes (DEGs).

Finally, a total of 890 DEGs were identified in the Os4_T samples. Of these genes, 399 were up-regulated and 491 were down-regulated in the Os4_T group compared with the Os4_C group (Fig. 1a). A heatmap was generated using hierarchical cluster analysis to show the expression of 20 DEGs which changed most significantly (Fig. 1b). Gene Ontology (GO) enrichment analysis was performed and we found that over 200 DEGs were involved in protein binding (Fig. 1d).

From these DEGs, we selected 10 up-regulated and 10 down-regulated genes based on the significance and GO enrichment analysis results. These genes were validated by quantitative real-time PCR (qPCR) (Fig. 1c).

\section{HSPB7 rescued the impaired osteogenesis of BMSC induced by TNF- $a$}

Among these selected genes, HSPB7 expression was reduced significantly in the Os4_T group. HSPB7 is mainly involved in protein binding and thus was selected as a potential therapeutic target. Western bolt assay has shown the decreased expression of HSPB7 in BMSCs treated with TNF-a for 4 days during osteogenesis (Fig. 2b-c).

From osteomyelitis patients and normal patients, the qPCR results showed that the mRNA level of HSPB7 was downregulated in osteomyelitis patients compared to that of normal patients, immunofluorescence (IF) showed that HSPB7 protein expression was significantly reduced in the bone peripheral muscle tissue of osteomyelitis patients (Supplementary Fig. 2b)

To clarify the protective role of HSPB7, we overexpressed HSPB7 in BMSCs by lentiviral vector transfection. Using IF, we chose 40 as the optimal multiplicity of infection (MOI), we performed western blot analyses to detect the expression of the osteo-specific proteins analyzed above, including Col1A1, Runx2, Osteoponitn (OPN) and SP7.

Compared with the control group, 4 days TNF-a $(100 \mathrm{ng} / \mathrm{mL})$ treatment significantly reduced the protein expression of Col1A1, Runx2, OPN and SP7, and the inhibitory effect of TNF-a was significantly reduced by HSPB7 Overexpression (OE). And HSPB7 overexpression also promoted the protein expression of Col1A1, OPN and SP7 compared with control group (Fig. 2d-e). Alizarin Red S (ARS) and ALP staining results showed that HSPB7 overexpression did not change the calcium deposit level nor ALP activity induced by TNF-a (Fig. 2g-h).

\section{HSPB7 protects the impaired osteogenesis induced by TNF- a via the CMA pathway}


To determine the mechanism underlying the protective role of HSPB7, we sought to identify its protein binding partners. Coomassie blue staining and silver staining confirmed that HSPB7 interacted with several proteins (Supplementary Fig. 3a-b), and a mass spectrometry assay was performed to identify these interactions. Mass spectrum results showed that HSPB7 interacted with heat shock protein 105 (HSPH1), which has been found to be in a complex with heat shock cognate A8 (HSCA8) and accelerates the ATP hydrolysis of HSCA8 ATPase(Hatayama et al., 1998; Yamagishi et al., 2000).

We then performed in vitro and in silico experiments to determine the nature of the interactions among HSPB7, HSCA8 and HSPH1. In vitro co-immunoprecipitation (CoIP) experiments performed using exogenous recombinant proteins showed that HSPH1 interacted with HSPB7 and HSCA8 (Fig. 3a, Supplementary Fig. 3c), but HSPB7 was not able to interact with HSCA8 (data didn't show here). When HSPB7, HSCA8 and HSPH1 were subjected to ColP together, HSPH1 would preferentially interact with HSPB7, however, HSPB7 was not able to interact with HSPH1 which interacted with HSCA8 (Fig. 3a, Supplementary Fig. 3c).

We then performed in vivo ColP experiments to confirm the interaction between HSPB7 and HSPH1 (Fig. 3b, Supplementary Fig. 3d). Since HSCA8 is one of the important components of chaperone-mediated autophagy, we performed an IF assay to detect the activity of chaperone-mediated autophagy in BMSCs treated with TNF- $a$ for $4 \mathrm{~d}$ during osteogenesis. The results showed that TNF- $a$ decreased the CMA activity, but HSPB7 overexpression significantly enhanced CMA activity inhibited by TNF-a (Fig. 3c).

Western blot assay showed that one day treatment of TNF-a $(1-100 \mathrm{ng} / \mathrm{mL})$ promoted the expression of LC3B and decreased the expression of SQSTM1/p62 on the first day in BMSCs of osteogenesis, but enhanced the expression of SQSTM1/p62 significantly when BMSCs were treated for four days (Fig. 3de). Additionally, we detected the activity of macroautophagy through Ad-GFP-LC3B and Ad-GFP-p62 infection, finding that macroautophagy influx decreased when treated with TNF-a for 4 days in BMSC during osteogenesis. HSPB7 overexpression didn't change the state of macroautophagy flux (Fig. $3 f-g$ ). HSPH1 gene expression increased when BMSCs were treated with TNF- $a(1-100 \mathrm{ng} / \mathrm{mL})$ more than $3 \mathrm{~d}(4-$ 6d) during osteogenesis differentiation (Supplementary Fig. 3e-f). HSPH1 gene depletion by specific interfering RNAs (siRNAs) promoted the expression of osteo-specific proteins including COL1A1 and RUNX2 in hBMSC (Fig. 4a-b), and HSPH1 depletion rescued the osteogenic differentiation impaired by TNF-a, however, HSCA8 or LAMP2A gene depletion attenuated the protect effect (Fig. 4c-f). Then we built Lamp2a overexpression hBMSCs using adeno-associated virus, and found Lamp2a overexpression rescued the impaired osteogenic differentiation of hBMSCs induced by TNF-a, and this effect was almost abrogated by DKK1, a specific Wnt/ß-catenin signaling pathway inhibitors (Fig. 4g-h).

\section{HSPB7 ${ }^{O E}$ MBMSCs protected fracture healing impaired by TNF-a in a murine tibial fracture model}

A murine tibial fracture model was used to investigate the protective effects of HSPB7 on TNF-a impaired osteogenesis in vivo. TNF- $\mathrm{a}(100 \mathrm{ng} / \mathrm{mL})$ was locally administered on the day of surgery and injected repeatedly on days 2, 4, and 6 after surgery, resulting in impaired fracture healing. Impaired fractured 
healing was rescued by the addition of HSPB7 overexpressing (OE) lentiviral particles. Animals that received HSPB7 overexpressing lentiviral particles (TNF- $a-B 7^{\mathrm{OE}}$ group) showed increased expression of RUNX2 and SP7 compared to the group received GFP lentiviral particles (TNF-a-GFP) (Fig. 5a-d). The number of Type $\mathrm{H}$ (CD31 ${ }^{\text {hi }} / \mathrm{Emcn}^{\text {hi }}$ ) endothelial cells, which are important for bone formation,(Kusumbe et al., 2014) also increased significantly in TNF-a-B7 ${ }^{O E}$ group compared to the TNF-a-GFP group (Fig. 5ef).

The fracture healing effect was also assessed by staining with the Masson's trichrome stain, Safranin 0 and fast green stain, which indicated the remodeling of the mineralized callus of the fracture healing samples at day 14 (Fig. 6a-b).

To confirm this observation quantitatively, fracture healing samples were collected at day 28 and analyzed by microcomputed tomography (microCT). The quantitative analysis of the micro-CT data further validated the histological results that HSPB7 protects the impaired fracture healing induced by TNF-a (Fig. 6c-e).

\section{Discussion}

Prolonged TNF-a stimulation impaires osteogenic differentiation of MSCs which is essential for fracture healing. Herein, we offer the first evidence that HSBP7 serves as a novel therapy target rescue the impaired osteogenesis of MSCs induced by TNF-a, wherein, chaperone-mediated autophagy plays an important role.

TNF-a was confirmed to have a major role in the inflammatory response during fracture healing, by recruiting MSCs and by promoting their differentiation. Previous studies have shown that treatment times of TNF-a have opposite effects on the osteogenesis of BMSCs. Here, we provide a general view of the effect of TNF-a on the osteogenesis of BMSCs over different periods of time. In the first early days (1-2d), TNF-a promoted the expression of osteo-related proteins of BMSCs during osteogenesis, however, the opposite effect was observed after $3 \mathrm{~d}$. Interestingly, TNF-a effectively enhanced calcium deposits formation and ALP activity regardless of the treatment time.

The mRNA microarray showed that endogenous HSPB7 expression was reduced during osteogenesis in a TNF-a dose-dependent manner. In sHSP family, HSPB1 and HSPB8 have been found to play an important role in bone metabolism. Unlike other sHSP members, HSPB7 is highly expressed in the heart and is one of the least studied. HSPB7 is also expressed in the skeletal muscle, however, no studies have explored the effect of HSPB7 in the skeletal system. Here, we found that HSPB7 effectively rescued the impaired osteogenesis of BMSCs caused by TNF-a treatment, meanwhile, it did not influence the promoting effect of TNF- $\alpha$ on the calcium deposits and ALP activity. In vivo, murine tibial fracture model shown that HSPB7 has a protective effect of on TNF-a impaired osteogenesis. Thus, HSPB7 may be a therapeutic target for impaired fracture healing caused by overdose TNF-a in disease including osteomyelitis and systemic lupus erythematosus. 
In human atherosclerotic vascular smooth cells, TNF-a promotes macroautophagy-related genes including microtubule-associated protein 1 light chain 3 (MAPLC-3) and Beclin-1 (Jia et al., 2006). Activation of the autophagy pathway by TNF-a reduced the production of reactive oxygen species (ROS) (Baregamian et al., 2009; Sivaprasad and Basu, 2008). TNF-a induced necroptosis in L929 cells, while, autophagy induced by TNF-a suppressed this necroptosis process by blocking the p38-NF-kB pathway (Ge et al., 2018; Ye et al., 2011). The evidence mentioned above confirmed that autophagy played an important role of in the protection of TNF-a. In our study, we found that TNF-a promoted macroautophagy of BMSCs during osteogenesis in early days, however, macroautohagy flux decreased when treated with TNF-a for $4 \mathrm{~d}$. HSPB7 interacted with HSPH1 which suppressed the ATPase activity of HSCA8. Inhibition of chaperone-mediated autophagy not only reduced the osteogenesis ability of BMSCs, but also suppressed the rescue effect by depleting HSPH1 on the impaired osteogenesis induced by TNF-a.

However, this study has some limitations that should be considered. First, CMA activity deletion inhibited ARS composites and ALP activity, which demonstrated that TNF-a promoted ARS composites and ALP activity in a CMA-independent way, which should be further investigated. Second, in our study, we found that HSPB7 protected the impaired fracture healing induced by TNF-a via the CMA pathway, however, limited by laboratory conditions, we did not develop gene-editing animal models to confirm our results. Finally, CMA promotes cell survival through degrading KFERQ-like motif proteins which are present in about $30 \%$ of cytosolic soluble proteins and providing alternative source of amino acids(Cuervo, 2010; Cuervo et al., 1995), in our study, we did not clear whether CMA degrades proteins that inhibited the osteogenesis of BMSCs or CMA protects the osteogenesis of BMSCs only by providing alternative amino acids.

\section{Conclusion}

Taken together, these findings indicate that a heat shock protein family network including HSPB7, HSPH1 and HSCA8 protects the impaired osteogenesis induced by TNF-a via the CMA/ $\beta$-catenin pathway (Fig. 7). And in vivo, HSPB7 overexpression lentiviral particles effectively protects the impaired fracture healing induced by TNF-a in a mouse tibia fracture healing model.

\section{Methods}

\section{Reagents}

hBMSCs were purchased from Cyagen Biosciences (Guangzhou, China). hBMSC growth medium were purchased from Cyagen Biosciences. Cells cultured in a atmosphere of $5 \% \mathrm{CO} 2$ at $37^{\circ} \mathrm{C}$. Osteogenic induction medium was prepared according to previous methods(Hang et al., 2019).

Recombinant human TNF alpha were purchased from novoprotein (shanghai, china, \#C008), Recombinant Human HSPB7 (R\&D Systems), recombinant Human HSCA8 (Cayman) and recombinant Human HSPH1/HSPH1 (Novus) were purchased from Univ Biological. 
Antibodies used for western blotting including Tublin (1:1000, Beyotime, shanghai, china), RUNX2 (1:1000, Beyotime), COL1A1 (1:1000, Beyotime), LAMP2a (1:1000, Beyotime), HSPH1 (1:1000, Beyotime), SP7/Osterix (1:1000, Abcam, shanghai, china), HSPB7 (1: 1000, Abcam), HSCA8 (1:1000, Abcam). Antibodies used for COIP included HSPB7 (5ug, rabbit, Abcam), HSPH1 (5ug, rabbit, Beyotime), HSCA8 (5ug, mouse, Abcam). Second antibodies including Alexa Fluor 488, Alexa Fluor 555 and Alexa Fluor 647 were purchased from Beyotime.

The siRNAs were purchased from Genepharma (Hangzhou, china).

hHSCA8 sense: GCAAAGAAUCAAGUUGCAATT

hHSCA8 antisense: UUGCAACUUGAUUCUUUGCTT

hLAMP2a sense: CCAUGGCACUGUGACAUAUTT

hLAMP2a antisense: AUAUGUCACAGUGCCAUGGTT

hHSPH1 sense: GCUCUACUUAGACUCAAUATT

hHSPH1 antisense: UAUUGAGUCUAAGUAGAGCTT

\section{mRNA microarray}

$4 \mathrm{~d}$ TNF-a (100 ng/mL)-treated (Os4_T) and control (Os4_C) osteogenic BMSCs were prepared for mRNA microarray. Total RNA was isolated using RNA Isolation reagent (Takara), and then purified using RNeasy Mini Kit (Qiagen, Germany). A RIN number of total RNA was checked to examine RNA integration using Agilent Bioanalyzer 2100 (Agilent technologies, US). Then qualified RNA samples of each group were utilized to synthesis biotinylated cRNA targets for the Human RNA 8*60K Expression Microarray (Agilent technologies, USA). Then biotinylated cRNA targets hybridized with the Microarray slides, after that, slides were scanned by an Agilent Microarray Scanner G5761A (Agilent technologies, US). Data were extracted by Feature Extraction software (version10.7.1.1, Agilent technologies, US) and the raw data were normalized through Quantile algorithm. The Human RNA Expression Microarray experiments were performed according to the protocol of Agilent technologies at LC Sciences Corporation(Hangzhou, China. Genes with pvalue $<0.05$ and fold change $\geq 1.2$ were selected for further analysis. GO/KEGG pathway enrichment analysis were done use Fisher's exact test of the target genes. Microarray data was submitted to GEO, GEO accession number: GSE 176086.

\section{Cell proliferation assay}

Cells were cultured in 96-well plate at a density of 5000 cells per well, CCK-8 (Beyotime) of 10\% was added into wells and incubated with cells for $2 \mathrm{~h}$ at $37^{\circ} \mathrm{C}$. Then the cell proliferation was measured by a microplate reader at the absorbance of $450 \mathrm{~nm}$ (BioTek, ELX808).

\section{Protein interaction assay}


For in vitro immunoprecipitation, recombinant proteins were incubated within lysis buffer contained protease inhibitors and phosphatase inhibitors for 20 minutes at RT. Then adding anti-rabbit HSPB7 or anti-rabbit HSPH1 incubated for 90 min at RT, rabbit IgG (A7016, Beyotime) was used as a negative control. Then the mixture was incubated with protein $\mathrm{G}$ gammabind plus sepharose (17088601, GE life, SWEDEN) for $2 \mathrm{~h}$ at RT. protein complex were centrifuged and washed with lysis buffer 3 times, suspended in $2 \mathrm{X}$ loading buffer and boiling for $5 \mathrm{~min}$ at $100^{\circ} \mathrm{C}$. Finally, proteins were detected by western blotting.

For in vivo co- immunoprecipitation assay, cells were lysed in lysis buffer contained protease inhibitors and phosphatase inhibitors for 30 min, centrifuged and the supernatant were cleared with protein $\mathrm{G}$ for $10 \mathrm{~min}$ at $4^{\circ} \mathrm{C}$. Then the cell lysates were incubated with primary antibodies and rabbit IgG which as a negative control overnight $(16 \mathrm{~h})$ at $4^{\circ} \mathrm{C}$. Then the antibodies were captured by protein $\mathrm{G}$ gammabind plus sepharose (17088601, GE life, SWEDEN) overnight at $4^{\circ} \mathrm{C}$. Protein complex were centrifuged and washed with lysis buffer 3 times, suspended in $2 X$ loading buffer and boiling for $5 \mathrm{~min}$ at $100^{\circ} \mathrm{C}$. Finally, proteins were detected by western blotting, coomassie blue staining and silver staining.

\section{Protein mass spectrometry analysis}

Proteins interacted with HSPB7 were freeze dried and enzymolysised by Trypsinbuffer for $16-18 \mathrm{~h}$ at $37^{\circ} \mathrm{C}$. Enzymolysis products were separated by high performance liquid chromatography (HPLC) according to the manufacturer's instructions. Then the enzymolysis products were analyzed by QExactive mass spectrometer (ThermoFisher). Finally, the RawFile of mass spectrum were analyzed by MaxQuant

\subsubsection{1 software.}

\section{RT-qPCR}

Total RNA from cells or human bone tissues were extracted with RNA isolation reagent (Takara) and RNA was quantified by spectrophotometer at $260 \mathrm{~nm}$ wave length (NanoDrop 2000; ThermoFisher). Total RNA was then reverse-transcribed with the Double-Strand cDNA Synthesis reagent (Takara) and cDNA (2 ul) was used to quantification using SYBR Green PCR Master Mix reagent (Takara), and finally detected by a StepOnePlus System (Applied Biosystems). The reaction conditions were as previous described.

\section{Western blot analysis}

Proteins was extracted from cells by lysing in RIPA buffer supplemented with protease inhibitors and phosphatase inhibitors (Beyotime). After protein quantification, cell lysates were suspended in 5X loading buffer and then equal amounts of proteins were subjected to migration on $10 \%-12 \%$ polyacrylamide gels, then transferred to a polyvinylidene fluoride membrane (Millipore, Shanghai, China). The membranes were blocked by non-fat milk (5\%) for $60 \mathrm{~min}$ and then incubated with primary antibodies for $12 \mathrm{~h}-16 \mathrm{~h}$ at $4^{\circ} \mathrm{C}$. After, membranes were washed with Tris-buffered saline-Tween (TBST) 3 times (10 min each) and incubated with HRP-conjugated secondary antibodies (Beyotime) for $1 \mathrm{~h}$ at RT. Finally, proteins were revealed by chemiluminescence reagents (Millipore) and the signal intensity was detected using Bio-Rad XRS system (Bio-Rad). 


\section{ALP staining}

Cells were passaged to 12-well plates and cultured with osteogenic induction medium when cells reached at $70 \%$ confluence. Osteogenic induction medium cultured for 5 days, cells were washed by PBS 3 times, and were fixed in $4 \%$ paraformaldehyde for $20 \mathrm{~min}-30 \mathrm{~min}$ at RT. Washed by PBS 3 times, cells were stained by the ALP Color Development Kit (Beyotime). Finally, the ALP activity quantification was performed as previous described.

\section{Alizarin Red staining}

Cells were passaged to 12-well plates and cultured with osteogenic induction medium when cells reached at $70 \%$ confluence. Osteogenic induction medium cultured for 15 days, cells were washed by PBS 3 times and were fixed in $4 \%$ paraformaldehyde for $20 \mathrm{~min}-30 \mathrm{~min}$ at RT. Washed by PBS for 3 times, calcium deposition was stained by Alizarin Red staining (Cyagen) for $5 \mathrm{~min}-10$ min at RT, and then was rinsed by PBS 3 times. The ARS quantification was performed as previous described.

\section{siRNA transfection}

Cells were seeded in plates and transfected with siRNA when cells reached $30-50 \%$ confluence. Transfection process was according to the manufacturer's instructions. Transfection efficiency was verified by qPCR.

\section{Adenovirus transfection}

Adenovirus expressing the GFP-LC3B (Ad-GFP-LC3B) and GFP-P62 (Ad-GFP-P62) were purchased from Beyotime. Briefly, cells were cultured in 12-well plates and transfected with adenovirus when cells reached at $70 \%$ confluence according to the manufacturer's instructions. Finally, signals were visualized by a fluorescence microscope (Leica) and quantified by Image $\mathrm{J}$ software.

\section{Lentiviral overexpression HSPB7}

Human and murine Lentiviral overexpression GFP-HSPB7 (GFP-B7 ${ }^{\mathrm{OE}}$ ) particles and the GFP control group (GFP-NC) were purchased from Cyagen Biosciences. A multiplicity of infection (MOI) which was used as the optimal amount of virus dose was confirmed by IF assay. 50-60\% confluent cells were incubated with lentiviral particles at a $\mathrm{MOl}$ of 40 and $4 \mu \mathrm{g} / \mathrm{ml}$ polybrene. $12 \mathrm{~h}$ later, the culture medium was changed and the transfected cells were used in next experiments.

\section{Immunofluorescence assay for CMA-active lysosomes}

CMA-active lysosomes were identified by the colocalization of LAMP-2A and HSCA8, procedures were performed according to the standard immunofluorescence protocol described by Cuervo et.(Kaushik and Cuervo, 2009). And signals were detected by a confocal microscopy (Leica TCS SP8, USA) and quantified by Image $J$ software.

\section{Animals}


All C57/B16 mice (male, 12 weeks) were purchased from SLAC Laboratory Animal Co (Shanghai, China). All of the animal experiments were approved by the Institutional Animal Care and Use Committee of the 2nd Affiliated Hospital, Zhejiang University (No: 2020 - 1035). All mice were randomly divided into control and experimental groups.

Sample sizes used at 6-8 animals per experimental procedure

Mice-fracture model

Mice-fracture model was modified on the basis of previous method(Harry et al., 2008). Briefly, mice anesthetized by intraperitoneally injection of $0.3 \%$ pentobarbital sodium $(30 \mathrm{mg} / \mathrm{kg}$ body). Exposed the right lower limb, made an incision lateral between the middle of tibia tuberosity and crest. A 0.38-mm diameter intramedullary fixation pin was then inserted into the tibia's medullary canal at the level of the tibia tuberosity for fixation. Separated the soft tissue carefully and stripped the periosteum above the crest of tibia. Then an osteotomy was created above the crest of tibia. The same leg was used in each group.

For in vivo study of HSPB7 protecitve effects, animals were divided into three groups. 20ul volume of TNF-a $(100 \mathrm{ng} / \mathrm{ml})$ or PBS was injected at the fracture site locally on days $0,2,4$ and 6 . GFP-B ${ }^{\mathrm{OE}}$ or GFP$\mathrm{NC}$ lentiviral particles were injected at day 6.2 and 4 weeks after surgery, limbs were harvested by lethal intraperitoneal injection of $0.1 \mathrm{ml}$ sodium pentobarbitone $(200 \mathrm{mg} / \mathrm{ml})$ for next experiments.

\section{Histology}

Following harvest, 2 weeks of samples were fixed by $10 \%$ paraformaldehyde for $36 \mathrm{~h}$ at $4^{\circ} \mathrm{C}$ and then were decalcified by $0.5 \mathrm{M}$ ethylene diaminetetra acetic acid (EDTA, Beyotime) for 3 days at $4^{\circ} \mathrm{C}$. Specimens were then embedded in paraffin and sectioned at a 5um thickness. Serial sections were deparaffinized and then stained with Safranin $O$ and Fast Green, Masson's Trichrome according to the standard procedures.

\section{Radiographic analysis}

Following harvest, 4 weeks of samples were send to make a microcomputed tomography $(\mu \mathrm{CT})$ evaluation. Each tibia was scanned using $\mu$ CT-100 imaging system (Scanco Medical, Switzerland), operation parameters were according to the previous report(Glass et al., 2011).

\section{Histology immunofluorescence analysis}

For mouse sample, following harvest, 2 weeks of samples were fixed by $10 \%$ paraformaldehyde for $4 \mathrm{~h}$ at $4^{\circ} \mathrm{C}$ and then were decalcified by 0.5M EDTA (Beyotime) for 3 days at $4^{\circ} \mathrm{C}$. Freshly dissected bone tissues and immunofluorescent stainings were according to previous methods described by Adams et(Kusumbe et al., 2014). The following primary antibodies were used: Sp7/Osterix (ab209484, abcam, diluted 1:100), Runx2 (ab192256, abcam, diluted 1:100), Endomucin (ab106100, abcam, diluted 1:100), CD31/PECAM (AF3628-SP, RD System, diluted 1:50). 
Then sections were blocked with 2\% BSA for 20 min at RT. Washed by PBS 3 times and incubated with primary antibody for $1 \mathrm{~h}$ at RT. Washed by PBS 3 times and incubated with second antibodies in a wet box for $1 \mathrm{~h}$ at RT. Then sections were washed by PBS 3 times and stained with DAPI. Target protein was observed under a fluorescence microscope (Leica) and quantified by Image $\mathrm{J}$ software.

\section{Statistical analysis}

All experiments were performed at least in triplicate, data are presented as means $\pm S D$, statistical significance between two groups was determined by Student's t test, one-way ANOVA or Bonferroni's posthoc test according to the distribution of the tested population. Statistical analysis was performed with SPSS 19.0 software (IBM, USA). A value of $P \leq 0.05$ was considered significant.

\section{Abbreviations}

HSPB7: heat shock protein family B (small) member 7

HSPH1: heat shock protein family $\mathrm{H}$ (small) member 1

HSCA8: heat shock cognate A8

CMA: chaperone-mediated autophagy

LAMP2A: lysosome-associated membrane protein 2A

BMSCs: Human bone marrow mesenchymal stem cells

sHSPs: Small heat shock proteins

ALP: alkaline phosphatase

ARS: Alizarin Red S

MOI: multiplicity of infection

\section{Declarations}

\section{Availability of data and materials}

The datasets used and/or analyzed during the current study are available from the corresponding author on reasonable request.

\section{Consent for publication}

All authors agree to publish this manuscript. 
The authors declare no conflicts of interest.

\section{Acknowledgments}

We thank all clients who have offered needing assistance for our study from clinical research center of the second affiliated hospital, Zhejiang university, including Xing Zhang, Meirong Yu, Yuelan Chen, Jiayu Yang, Am Liu and Chunc Li et al. We also appreciate the general help of LL Zhang from orthopedic research center of the second affiliated hospital, Zhejiang university.

\section{Authors' Contributions}

D. T. Xue and Z. Pan designed the research; K H, LY and Jw B performed the experiments; Zh K, Jx X, Yb W and $\mathrm{Cw} Z$ analyzed the data; and K H, D. Xue, and Z. Pan wrote the paper.

\section{Funding}

This work was supported by grants from the National Natural Science Foundation of China (Nos. 81672147; Nos. 81874007).

\section{Ethics approval and consent to participate}

All animal experiments were in accordance to the Animal Care and Use Committee guidelines of Zhejiang University. All experimental procedures were in accordance with the and Institutional Animal Care Use Committee at Zhejiang University. The human samples obtained procedure was approved by the ethics committee of the second affiliated hospital of Zhejiang University.

\section{References}

1. Ai-Aql, Z.S., Alagl, A.S., Graves, D.T., Gerstenfeld, L.C., and Einhorn, T.A. (2008). Molecular mechanisms controlling bone formation during fracture healing and distraction osteogenesis. Journal of dental research 87, 107-118. 10.1177/154405910808700215.

2. Al-Sebaei, M.O., Daukss, D.M., Belkina, A.C., Kakar, S., Wigner, N.A., Cusher, D., Graves, D., Einhorn, T., Morgan, E., and Gerstenfeld, L.C. (2014). Role of Fas and Treg cells in fracture healing as characterized in the fas-deficient (Ipr) mouse model of lupus. Journal of bone and mineral research : the official journal of the American Society for Bone and Mineral Research 29, 1478-1491. 10.1002/jbmr.2169.

3. Alblowi, J., Kayal, R.A., Siqueira, M., McKenzie, E., Krothapalli, N., McLean, J., Conn, J., Nikolajczyk, B., Einhorn, T.A., Gerstenfeld, L., and Graves, D.T. (2009). High levels of tumor necrosis factor-alpha contribute to accelerated loss of cartilage in diabetic fracture healing. The American journal of pathology 175, 1574-1585. 10.2353/ajpath.2009.090148.

4. Baregamian, N., Song, J., Bailey, C.E., Papaconstantinou, J., Evers, B.M., and Chung, D.H. (2009). Tumor necrosis factor-alpha and apoptosis signal-regulating kinase 1 control reactive oxygen 
species release, mitochondrial autophagy, and c-Jun N-terminal kinase/p38 phosphorylation during necrotizing enterocolitis. Oxidative medicine and cellular longevity 2, 297-306.

10.4161/oxim.2.5.9541.

5. Boregowda, S.V., Krishnappa, V., and Phinney, D.G. (2016). Isolation of Mouse Bone Marrow Mesenchymal Stem Cells. Methods in molecular biology (Clifton, N.J.) 1416, 205-223. 10.1007/9781-4939-3584-0_11.

6. Cho, H.H., Kyoung, K.M., Seo, M.J., Kim, Y.J., Bae, Y.C., and Jung, J.S. (2006). Overexpression of CXCR4 increases migration and proliferation of human adipose tissue stromal cells. Stem cells and development 15, 853-864. 10.1089/scd.2006.15.853.

7. Claes, L., Recknagel, S., and Ignatius, A. (2012). Fracture healing under healthy and inflammatory conditions. Nature reviews. Rheumatology 8, 133-143. 10.1038/nrrheum.2012.1.

8. Cottrell, J., and O'Connor, J.P. (2010). Effect of Non-Steroidal Anti-Inflammatory Drugs on Bone Healing. Pharmaceuticals (Basel, Switzerland) 3, 1668-1693. 10.3390/ph3051668.

9. Cuervo, A.M. (2010). Chaperone-mediated autophagy: selectivity pays off. Trends Endocrinol Metab 21, 142-150. 10.1016/j.tem.2009.10.003.

10. Cuervo, A.M., Knecht, E., Terlecky, S.R., and Dice, J.F. (1995). Activation of a selective pathway of lysosomal proteolysis in rat liver by prolonged starvation. The American journal of physiology 269, C1200-1208. 10.1152/ajpcell.1995.269.5.C1200.

11. Cuervo, A.M., and Wong, E. (2014). Chaperone-mediated autophagy: roles in disease and aging. Cell research 24, 92-104. 10.1038/cr.2013.153.

12. Dice, J.F. (1990). Peptide sequences that target cytosolic proteins for lysosomal proteolysis. Trends in biochemical sciences 15, 305-309. 10.1016/0968-0004(90)90019-8.

13. Ding, J., Ghali, O., Lencel, P., Broux, O., Chauveau, C., Devedjian, J.C., Hardouin, P., and Magne, D. (2009). TNF-alpha and IL-1beta inhibit RUNX2 and collagen expression but increase alkaline phosphatase activity and mineralization in human mesenchymal stem cells. Life sciences $84,499-$ 504. 10.1016/j.lfs.2009.01.013.

14. Ge, Y., Huang, M., and Yao, Y.M. (2018). Autophagy and proinflammatory cytokines: Interactions and clinical implications. Cytokine \& growth factor reviews 43, 38-46. 10.1016/j.cytogfr.2018.07.001.

15. Gerstenfeld, L.C., Cho, T.J., Kon, T., Aizawa, T., Tsay, A., Fitch, J., Barnes, G.L., Graves, D.T., and Einhorn, T.A. (2003a). Impaired fracture healing in the absence of TNF-alpha signaling: the role of TNF-alpha in endochondral cartilage resorption. Journal of bone and mineral research : the official journal of the American Society for Bone and Mineral Research 18, 1584-1592. 10.1359/jbmr.2003.18.9.1584.

16. Gerstenfeld, L.C., Cullinane, D.M., Barnes, G.L., Graves, D.T., and Einhorn, T.A. (2003b). Fracture healing as a post-natal developmental process: molecular, spatial, and temporal aspects of its regulation. Journal of cellular biochemistry 88, 873-884. 10.1002/jcb.10435.

17. Glass, G.E., Chan, J.K., Freidin, A., Feldmann, M., Horwood, N.J., and Nanchahal, J. (2011). TNF-alpha promotes fracture repair by augmenting the recruitment and differentiation of muscle-derived 
stromal cells. Proceedings of the National Academy of Sciences of the United States of America 108, 1585-1590. 10.1073/pnas.1018501108.

18. Hang, K., Ye, C., Xu, J., Chen, E., Wang, C., Zhang, W., Ni, L., Kuang, Z., Ying, L., Xue, D., and Pan, Z. (2019). Apelin enhances the osteogenic differentiation of human bone marrow mesenchymal stem cells partly through Wnt/beta-catenin signaling pathway. Stem cell research \& therapy 10,189 . 10.1186/s13287-019-1286-x.

19. Harry, L.E., Sandison, A., Paleolog, E.M., Hansen, U., Pearse, M.F., and Nanchahal, J. (2008). Comparison of the healing of open tibial fractures covered with either muscle or fasciocutaneous tissue in a murine model. Journal of orthopaedic research : official publication of the Orthopaedic Research Society 26, 1238-1244. 10.1002/jor.20649.

20. Hatayama, T., Yasuda, K., and Yasuda, K. (1998). Association of HSP105 with HSC70 in high molecular mass complexes in mouse FM3A cells. Biochemical and biophysical research communications 248, 395-401. 10.1006/bbrc.1998.8979.

21. Hess, K., Ushmorov, A., Fiedler, J., Brenner, R.E., and Wirth, T. (2009). TNFalpha promotes osteogenic differentiation of human mesenchymal stem cells by triggering the NF-kappaB signaling pathway. Bone 45, 367-376. 10.1016/j.bone.2009.04.252.

22. Huang, H., Zhao, N., Xu, X., Xu, Y., Li, S., Zhang, J., and Yang, P. (2011). Dose-specific effects of tumor necrosis factor alpha on osteogenic differentiation of mesenchymal stem cells. Cell proliferation 44 , 420-427. 10.1111/j.1365-2184.2011.00769.x.

23. Jia, G., Cheng, G., Gangahar, D.M., and Agrawal, D.K. (2006). Insulin-like growth factor-1 and TNFalpha regulate autophagy through c-jun $\mathrm{N}$-terminal kinase and Akt pathways in human atherosclerotic vascular smooth cells. Immunology and cell biology 84, 448-454. 10.1111/j.14401711.2006.01454.x.

24. Kappé, G., Franck, E., Verschuure, P., Boelens, W.C., Leunissen, J.A., and de Jong, W.W. (2003). The human genome encodes 10 alpha-crystallin-related small heat shock proteins: HspB1-10. Cell stress \& chaperones 8, 53-61. 10.1379/1466-1268(2003)8<53:thgecs>2.0.co;2.

25. Kaushik, S., and Cuervo, A.M. (2009). Methods to monitor chaperone-mediated autophagy. Methods in enzymology 452, 297-324. 10.1016/s0076-6879(08)03619-7.

26. Kaushik, S., and Cuervo, A.M. (2018). The coming of age of chaperone-mediated autophagy. Nature reviews. Molecular cell biology 19, 365-381. 10.1038/s41580-018-0001-6.

27. Kayal, R.A., Siqueira, M., Alblowi, J., McLean, J., Krothapalli, N., Faibish, D., Einhorn, T.A., Gerstenfeld, L.C., and Graves, D.T. (2010). TNF-alpha mediates diabetes-enhanced chondrocyte apoptosis during fracture healing and stimulates chondrocyte apoptosis through FOXO1. Journal of bone and mineral research : the official journal of the American Society for Bone and Mineral Research 25, 1604-1615. 10.1002/jbmr.59.

28. Ke, L., Meijering, R.A., Hoogstra-Berends, F., Mackovicova, K., Vos, M.J., Van Gelder, I.C., Henning, R.H., Kampinga, H.H., and Brundel, B.J. (2011). HSPB1, HSPB6, HSPB7 and HSPB8 protect against RhoA 
GTPase-induced remodeling in tachypaced atrial myocytes. PloS one 6, e20395.

10.1371/journal.pone.0020395.

29. Kon, T., Cho, T.J., Aizawa, T., Yamazaki, M., Nooh, N., Graves, D., Gerstenfeld, L.C., and Einhorn, T.A. (2001). Expression of osteoprotegerin, receptor activator of NF-kappaB ligand (osteoprotegerin ligand) and related proinflammatory cytokines during fracture healing. Journal of bone and mineral research : the official journal of the American Society for Bone and Mineral Research 16, 1004-1014. 10.1359/jbmr.2001.16.6.1004.

30. Kovach, T.K., Dighe, A.S., Lobo, P.I., and Cui, Q. (2015). Interactions between MSCs and immune cells: implications for bone healing. Journal of immunology research $2015,752510$.

10.1155/2015/752510.

31. Krief, S., Faivre, J.F., Robert, P., Le Douarin, B., Brument-Larignon, N., Lefrère, I., Bouzyk, M.M., Anderson, K.M., Greller, L.D., Tobin, F.L., et al. (1999). Identification and characterization of cvHsp. A novel human small stress protein selectively expressed in cardiovascular and insulin-sensitive tissues. J Biol Chem 274, 36592-36600. 10.1074/jbc.274.51.36592.

32. Kusumbe, A.P., Ramasamy, S.K., and Adams, R.H. (2014). Coupling of angiogenesis and osteogenesis by a specific vessel subtype in bone. Nature 507, 323-328. 10.1038/nature13145.

33. Lacey, D.C., Simmons, P.J., Graves, S.E., and Hamilton, J.A. (2009). Proinflammatory cytokines inhibit osteogenic differentiation from stem cells: implications for bone repair during inflammation. Osteoarthritis and cartilage 17, 735-742. 10.1016/j.joca.2008.11.011.

34. Liao, W.C., Juo, L.Y., Shih, Y.L., Chen, Y.H., and Yan, Y.T. (2017). HSPB7 prevents cardiac conduction system defect through maintaining intercalated disc integrity. PLoS genetics 13, e1006984. 10.1371/journal.pgen.1006984.

35. Loi, F., Córdova, L.A., Pajarinen, J., Lin, T.H., Yao, Z., and Goodman, S.B. (2016). Inflammation, fracture and bone repair. Bone 86, 119-130. 10.1016/j.bone.2016.02.020.

36. Lu, Z., Wang, G., Dunstan, C.R., and Zreiqat, H. (2012). Short-term exposure to tumor necrosis factoralpha enables human osteoblasts to direct adipose tissue-derived mesenchymal stem cells into osteogenic differentiation. Stem cells and development 21, 2420-2429. 10.1089/scd.2011.0589.

37. Maaroufi, H., and Tanguay, R.M. (2013). Analysis and phylogeny of small heat shock proteins from marine viruses and their cyanobacteria host. PloS one 8, e81207. 10.1371/journal.pone.0081207.

38. Marsell, R., and Einhorn, T.A. (2011). The biology of fracture healing. Injury 42, 551-555. 10.1016/j.injury.2011.03.031.

39. Mercer, E.J., Lin, Y.F., Cohen-Gould, L., and Evans, T. (2018). Hspb7 is a cardioprotective chaperone facilitating sarcomeric proteostasis. Developmental biology 435, 41-55.

10.1016/j.ydbio.2018.01.005.

40. Mymrikov, E.V., Seit-Nebi, A.S., and Gusev, N.B. (2011). Large potentials of small heat shock proteins. Physiological reviews 91, 1123-1159. 10.1152/physrev.00023.2010.

41. Shakoori, A.R., Oberdorf, A.M., Owen, T.A., Weber, L.A., Hickey, E., Stein, J.L., Lian, J.B., and Stein, G.S. (1992). Expression of heat shock genes during differentiation of mammalian osteoblasts and 
promyelocytic leukemia cells. Journal of cellular biochemistry 48, 277-287. 10.1002/jcb.240480308.

42. Sivaprasad, U., and Basu, A. (2008). Inhibition of ERK attenuates autophagy and potentiates tumour necrosis factor-alpha-induced cell death in MCF-7 cells. Journal of cellular and molecular medicine 12, 1265-1271. 10.1111/j.1582-4934.2008.00282.x.

43. Sun, Y., and MacRae, T.H. (2005). The small heat shock proteins and their role in human disease. The FEBS journal 272, 2613-2627. 10.1111/j.1742-4658.2005.04708.x.

44. Takayanagi, H. (2009). Osteoimmunology and the effects of the immune system on bone. Nature reviews. Rheumatology 5, 667-676. 10.1038/nrrheum.2009.217.

45. Vos, M.J., Zijlstra, M.P., Kanon, B., van Waarde-Verhagen, M.A., Brunt, E.R., Oosterveld-Hut, H.M., Carra, S., Sibon, O.C., and Kampinga, H.H. (2010). HSPB7 is the most potent polyQ aggregation suppressor within the HSPB family of molecular chaperones. Human molecular genetics 19, 4677-4693. $10.1093 / \mathrm{hmg} / \mathrm{ddq} 398$.

46. Wu, T., Mu, Y., Bogomolovas, J., Fang, X., Veevers, J., Nowak, R.B., Pappas, C.T., Gregorio, C.C., Evans, S.M., Fowler, V.M., and Chen, J. (2017). HSPB7 is indispensable for heart development by modulating actin filament assembly. Proceedings of the National Academy of Sciences of the United States of America 114, 11956-11961. 10.1073/pnas.1713763114.

47. Yamagishi, N., Nishihori, H., Ishihara, K., Ohtsuka, K., and Hatayama, T. (2000). Modulation of the chaperone activities of Hsc70/Hsp40 by Hsp105alpha and Hsp105beta. Biochemical and biophysical research communications 272, 850-855. 10.1006/bbrc.2000.2864.

48. Ye, Y.C., Yu, L., Wang, H.J., Tashiro, S., Onodera, S., and Ikejima, T. (2011). TNFa-induced necroptosis and autophagy via supression of the p38-NF-kB survival pathway in L929 cells. Journal of pharmacological sciences 117, 160-169. 10.1254/jphs.11105fp.

\section{Figures}




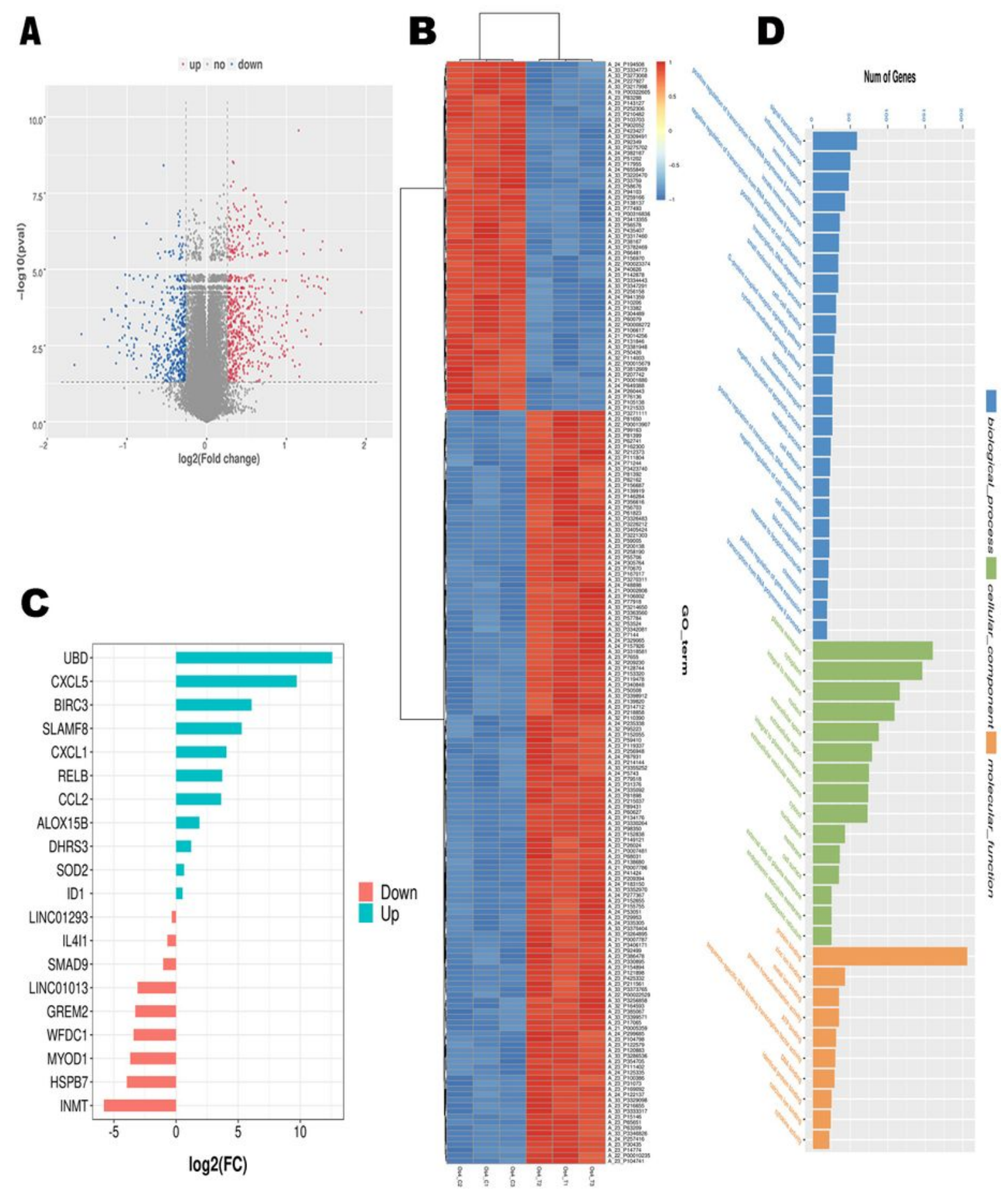

\section{Figure 1}

TNF-a treatment results in 890 differentially expressed genes. A) Volcano plot compared the expression (fold change) of mRNA between Os4_T and Os4_C; B) A heatmap of 20 DEGs which changed most significantly was generated using hierarchical cluster analysis; C) 20 selected DEGs are verified by qPCR; D) GO analysis of DEGs in Os4_T and Os4_C. 
A

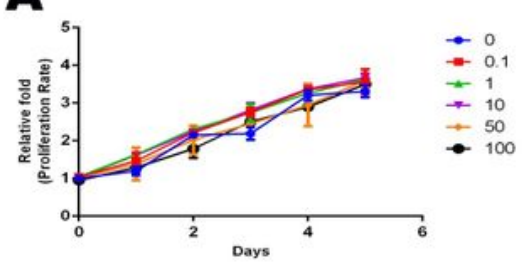

B

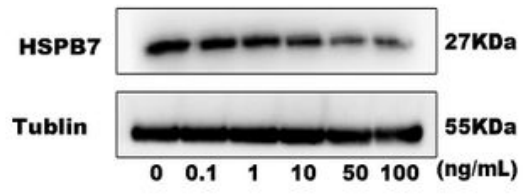

C

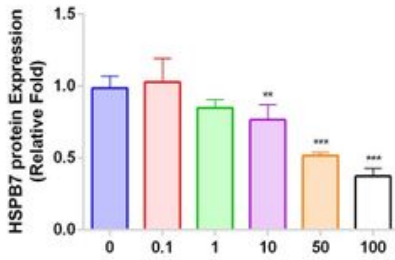

D

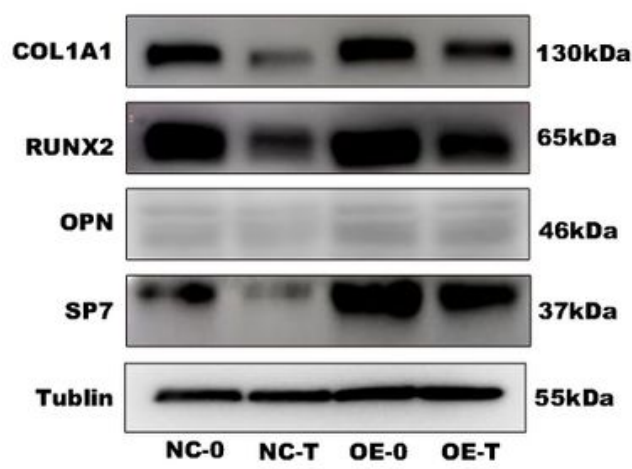

F
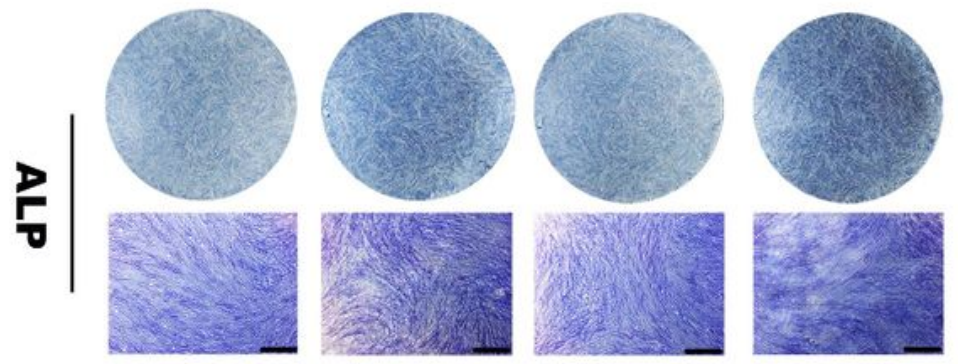

G
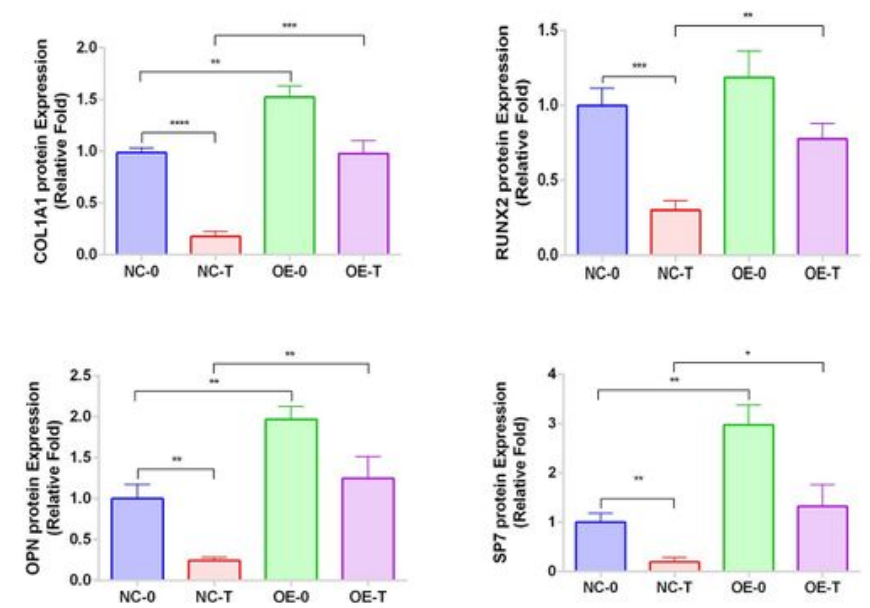

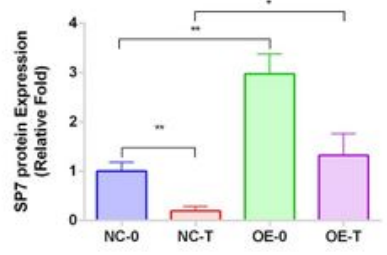

G

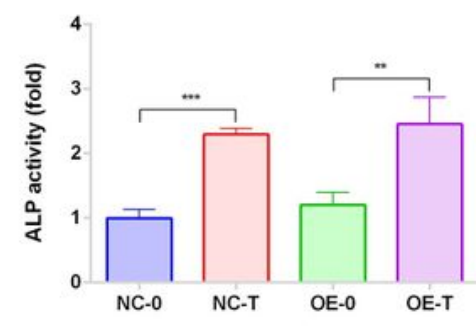

I

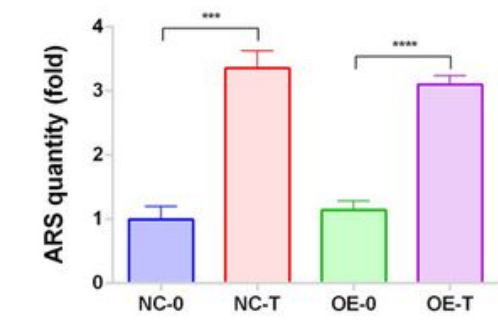

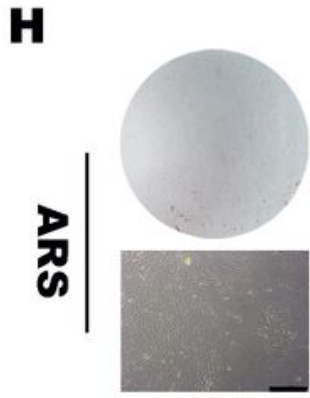

NC-O

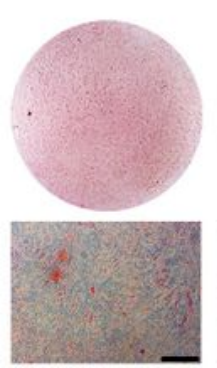

NC-T

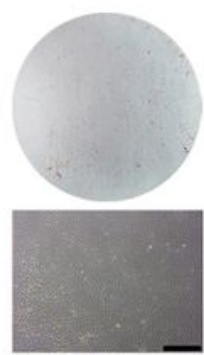

OE-0

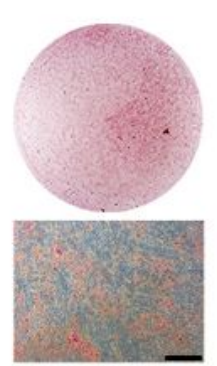

OE-T

\section{Figure 2}

HSPB7 overexpression rescued the impaired osteogenesis of BMSC induced by TNF-a. A) CCK-8 assay showed that no significant differences on cell number between different TNF-a concentration groups in the first 6 days; B-C) HSPB7 expression decreased in BMSCs treated with TNF- $a(0-100 \mathrm{ng} / \mathrm{mL})$ for 4 days during osteogenesis; D-E) Relative expression of osteo-specific proteins (RUNX2, COL1A1, OPN and SP7) on day 4 of osteogenesis, Protein expression levels were normalized to that of Tublin. OE: HSPB7 
overexpression, NC: HSPB7 overexpression control; F-G) Alizarin Red S (ARS) and alkaline phosphatase (ALP) activity staining. ALP activity staining was performed when cells were cultured for 5 days, ARS staining was performed when cells were cultured for 15 days. Scale bars, 500 um. Data are expressed as mean $\pm S D$. Assays were performed in triplicates. ${ }^{*}, P<0.05, * *, P<0.01, * \star *, P<0.001$ compared with the control group.

A

\begin{tabular}{|c|c|c|c|c|c|c|c|c|c|c|c|c|}
\hline \multirow{3}{*}{$\begin{array}{r}\text { HSP105 } \\
\text { HSC70 }\end{array}$} & PSPB7 & $\lg G$ & IP HSP105 & $\lg 6$ & IP HSP105 & IgG & \multirow{3}{*}{$\begin{array}{l}105 \mathrm{KDa} \\
70 \mathrm{KDa}\end{array}$} & \multirow{3}{*}{$\begin{array}{r}\text { HSP105 } \\
\text { HSPB7 }\end{array}$} & Input & IP HSPB7 & $\lg G$ & \multirow[b]{2}{*}{ 105KDa } \\
\hline & $=$ & & $\mathbf{E}$ & & e. & & & & - & $1 \cdots$ & & \\
\hline & & & & & & & & & 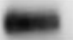 & & & 70KDa \\
\hline HSPB7 & 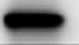 & $+\infty$ & - & $+\cdots$ & - & $=$ & $27 \mathrm{KDa}$ & Tublin & & & & 55KDa \\
\hline
\end{tabular}

C
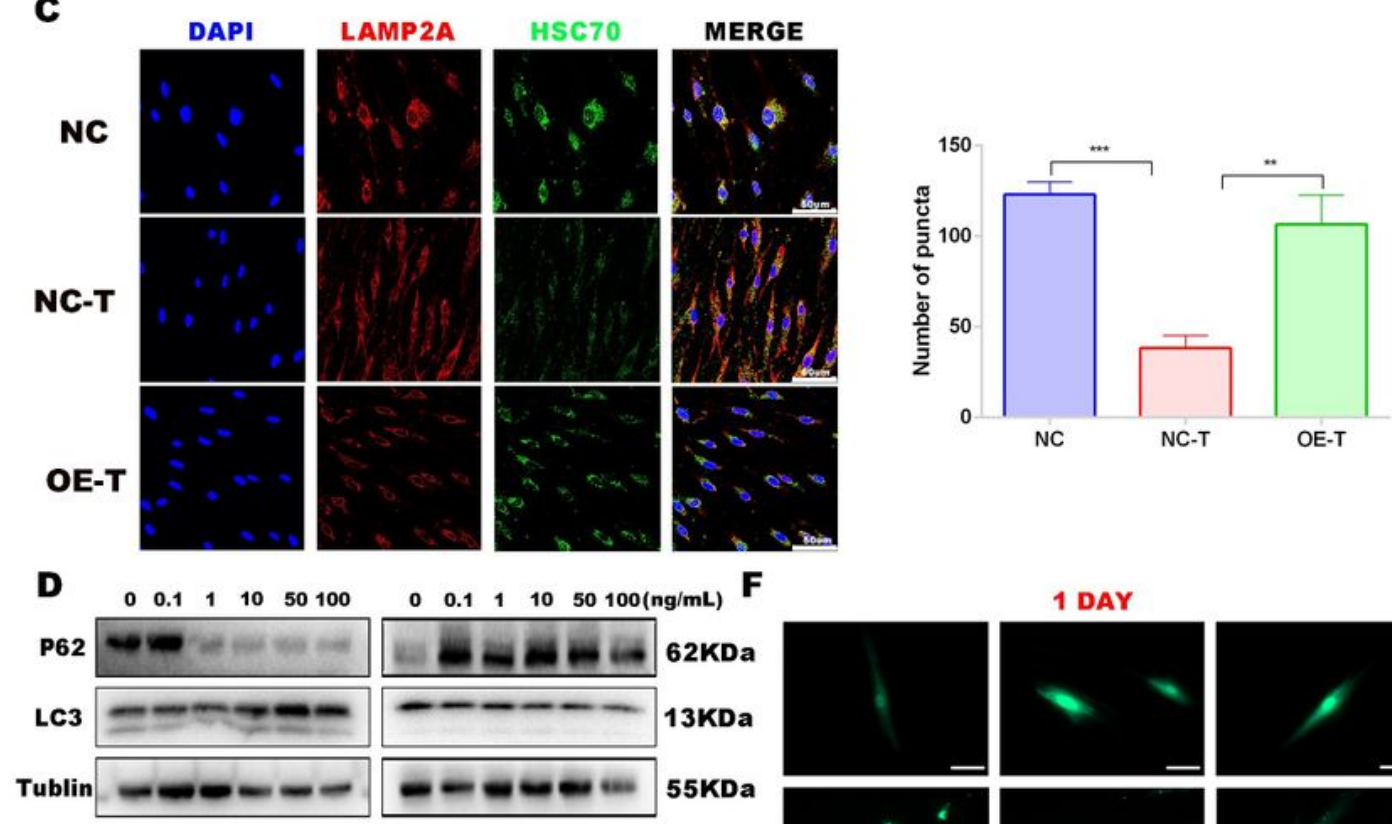

$\mathbf{E}$
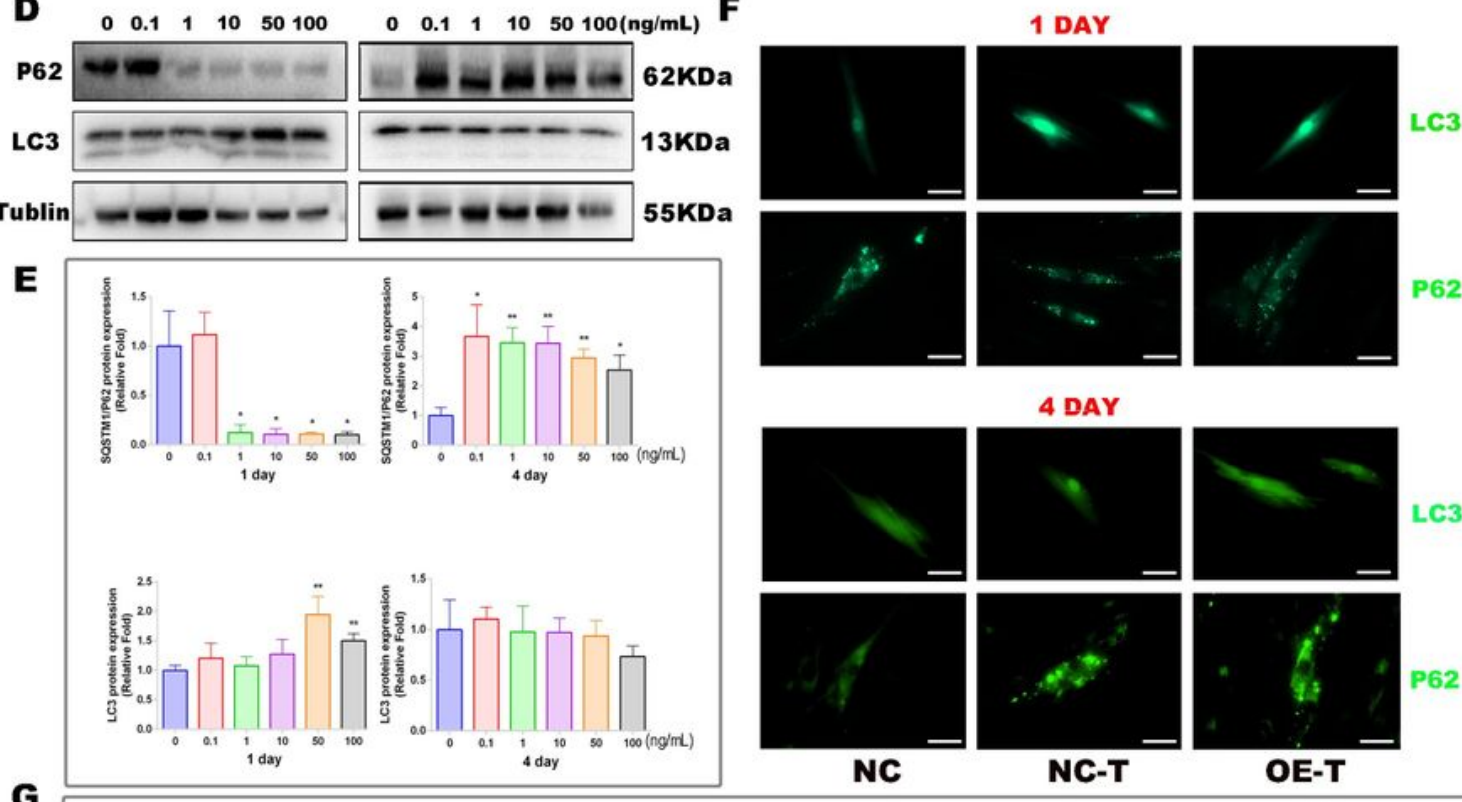

4 DAY
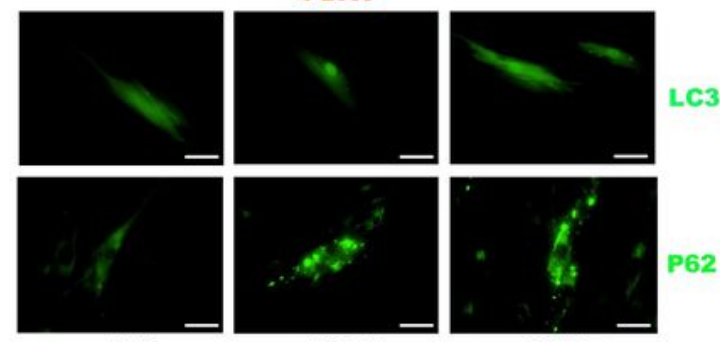

G
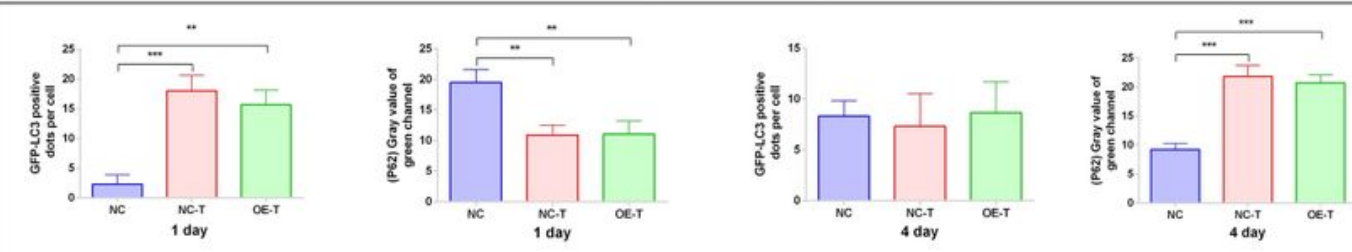

Figure 3 
HSPB7 overexpression rescued the impaired osteogenesis of BMSCs induced by TNF-a through CMA pathway. A) In vitro protein interaction assay showed that HSPH1 interacted with HSPB7 and HSCA8. HSPB7 was not able to interact with HSPH1 which interacted with HSCA8. B) In vivo ColP experiments confirmed the interaction between HSPB7 and HSPH1. C) IF showed that TNF-a decreased the CMA activity, HSPB7 overexpression significantly enhanced CMA activity inhibited by TNF-a. Scale bars, 50 um. Quantification of the average number of fluorescent puncta per cell. $\mathrm{N} \varangle 50$ cells. D-E) TNF-a (1-100 $\mathrm{ng} / \mathrm{mL}$ ) promoted the expression of LC3B and decreased the expression of SQSTM1/p62 on the first day in BMSCs of osteogenesis, but enhanced the expression of SQSTM1/p62 significantly when BMSCs were treated for four days. F-G) IF showed that macroautophagy influx decreased when treated with TNF-a for 4 days in BMSC during osteogenesis. HSPB7 overexpression didn't change the state of macroautophagy flux. Scale bars, 50 um. OE: HSPB7 overexpression, NC: HSPB7 overexpression control; Quantification of the average number of fluorescent puncta per cell. $\mathrm{N} \varangle 30$ cells. Data are expressed as mean $\pm S D$. Assays were performed in triplicates. ${ }^{*}, \mathrm{P}<0.05, * \star, \mathrm{P}<0.01$, $* \star \star, P<0.001$ compared with the control group. 


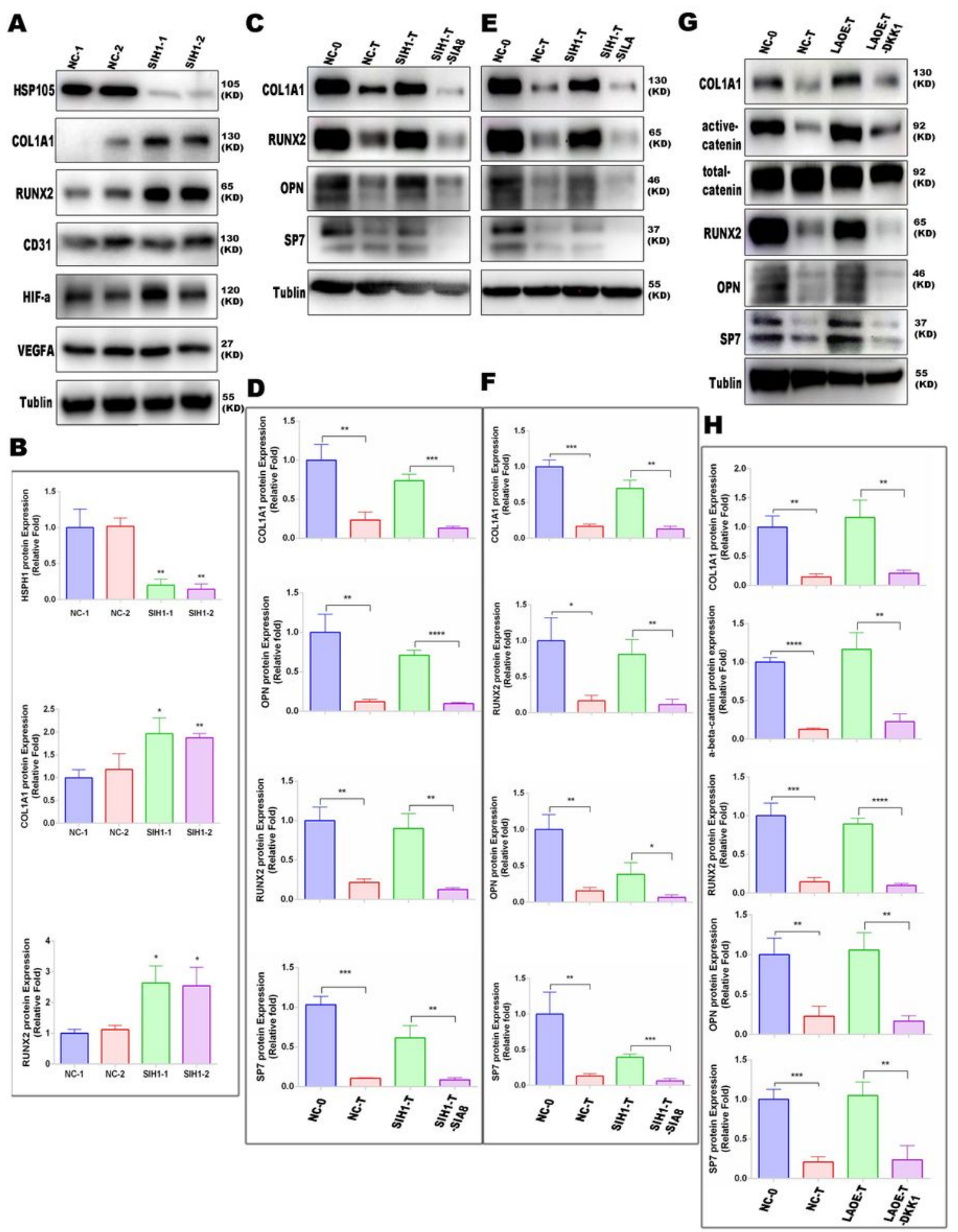

Figure 4

HSPB7 overexpression activating CMA pathway to protect the impaired osteogenesis of BMSCs induced by TNF-a through inhibiting HSPH1. A-B) The inhibition efficiency of siRNA was confirmed by western bolt; HSPH1 silencing significantly promoted the osteogenesis of BMSCs compared to the control group. HSPH1 silencing promoted the retention of HIF-1a, but have not effects on the expression of CD31 and VEGFA. C-F) HSPH1 silencing significantly reversed the impaired osteogenesis of BMSCs treated with 
TNF-a compared to the control group, and HSCA8 or LAMP2A silencing almost abrogated this protective effect against TNF-a. G-H) Lamp2a overexpression rescued the impaired osteogenic differentiation of hBMSCs induced by TNF-a, and this effect was almost abrogated by DKK1. Data are expressed as mean $\pm S D$. Assays were performed in triplicates. ${ }^{*}, P<0.05, * *, P<0.01$, $* * *, P<0.001$ compared with the control group.

A

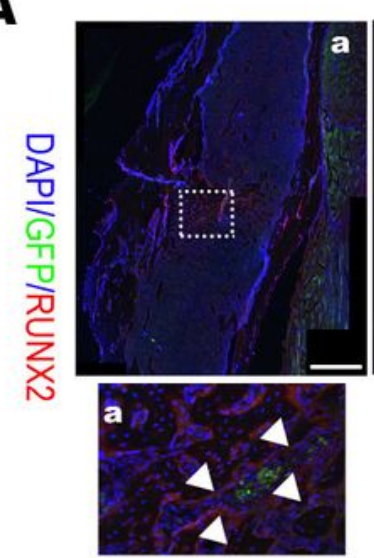

C
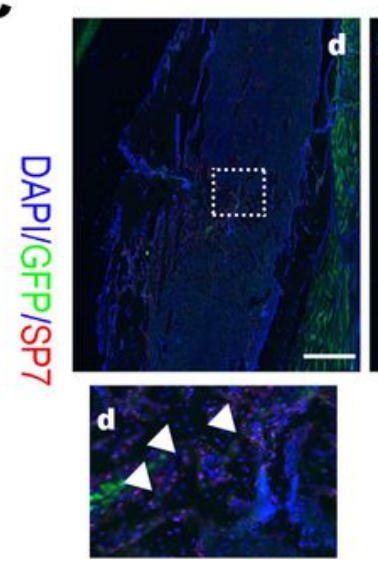

$\mathbf{E}$

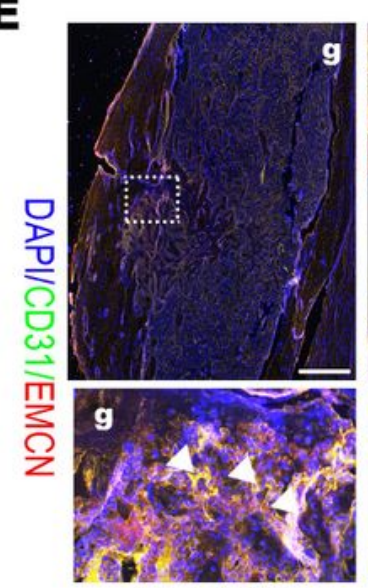

GFP-NC
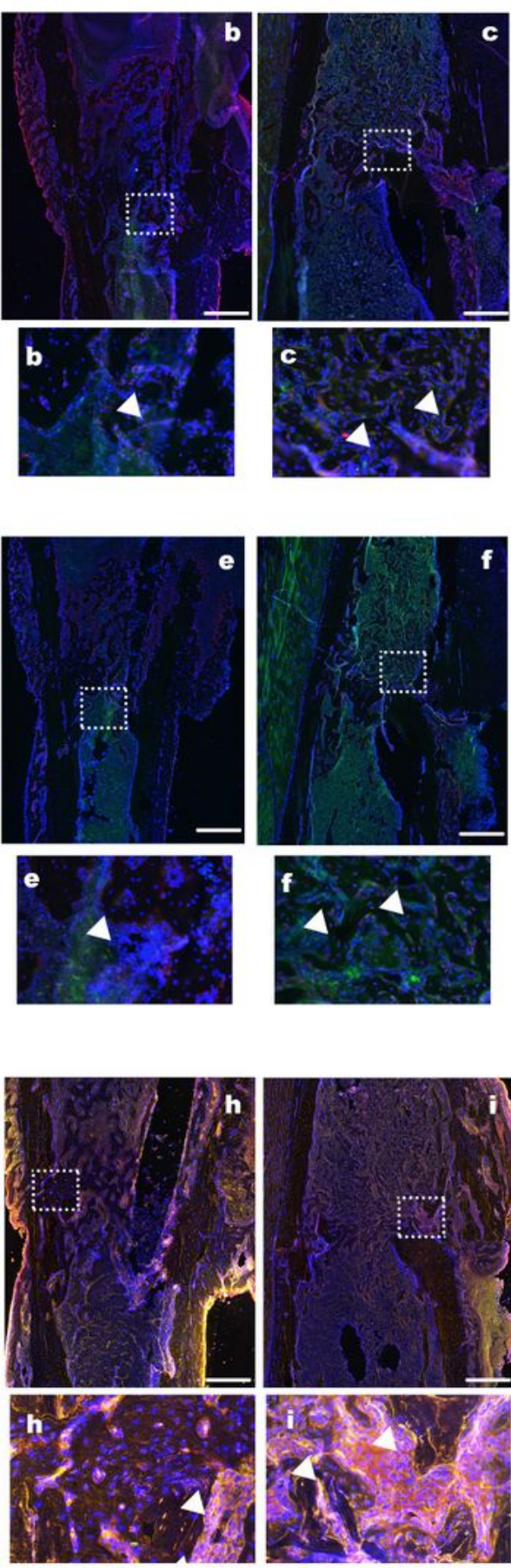

GFP-TNFa
B

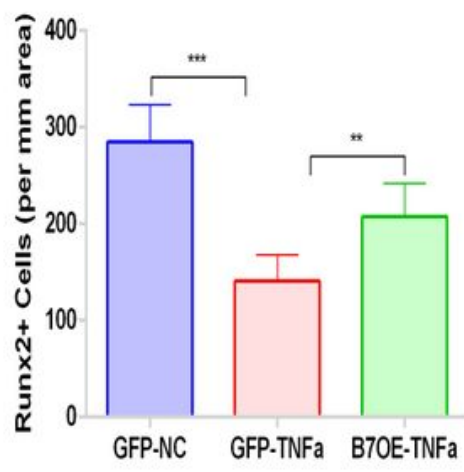

D

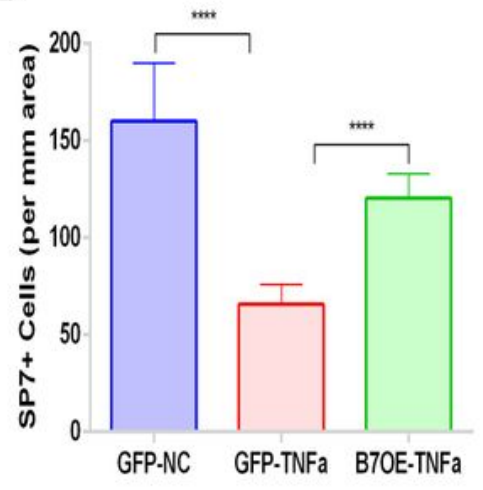

F

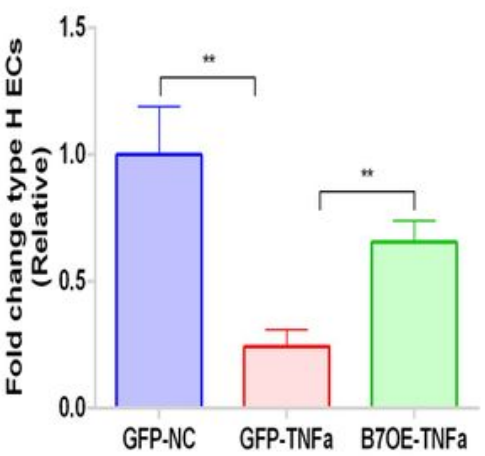

Figure 5 
GFP-B70E lentiviral particles rescued the Runx2 and SP7 expression and type $\mathrm{H}$ vessels invasion in a murine tibial fracture model. A-D) Quantitation of Runx2+ and SP7+ (Osx) cells. GFP-B70E lentiviral particles rescued the RUNX2 and SP7 expression impaired by TNF- $a(100 \mathrm{ng} / \mathrm{mL}),(\mathrm{n}=6$ mice in two independent experiments). Scale bars, 500 um. E-F) GFP-B7OE lentiviral particles rescued the type $\mathrm{H}$ vessels invasion impaired by TNF-a. Scale bars, 500 um. GFP-NC: GFP expressed lentiviral particles (Negative Control); GFP-TNF-a: GFP expressed lentiviral particles with TNF-a; B7OE-TNF-a: HSPB7

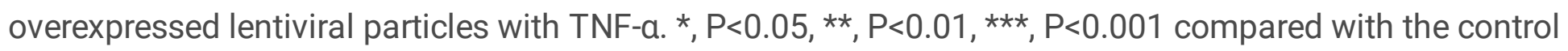
group. 
A
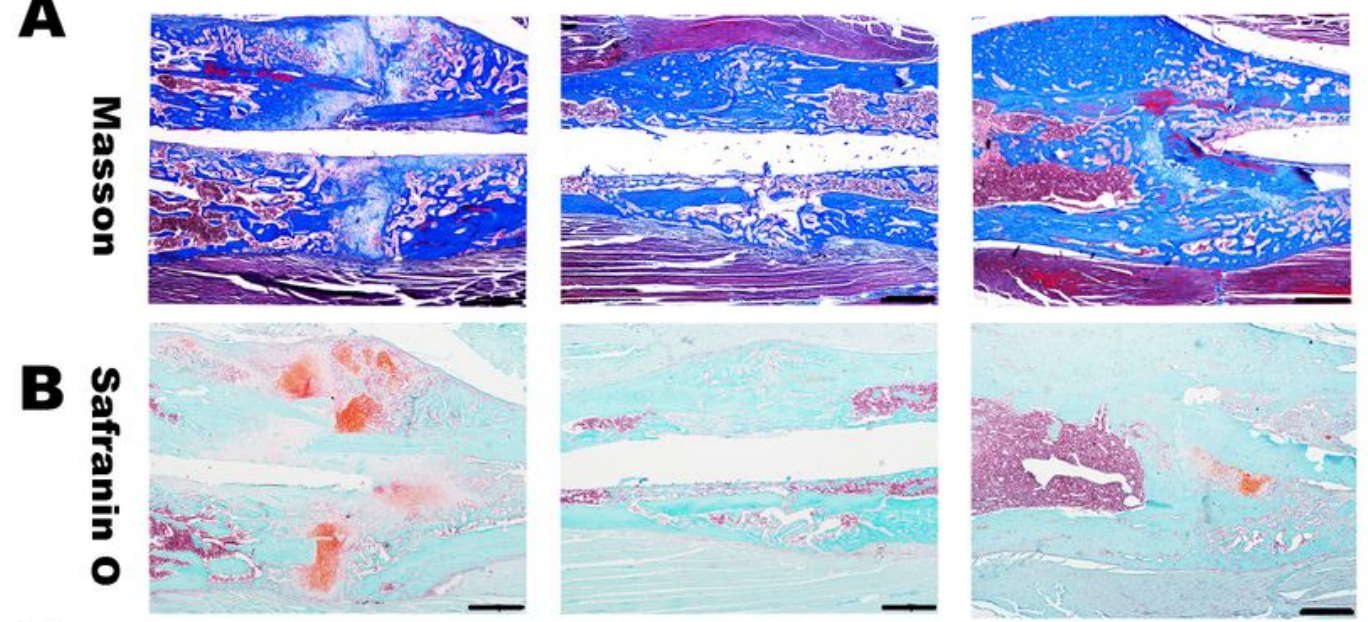

C
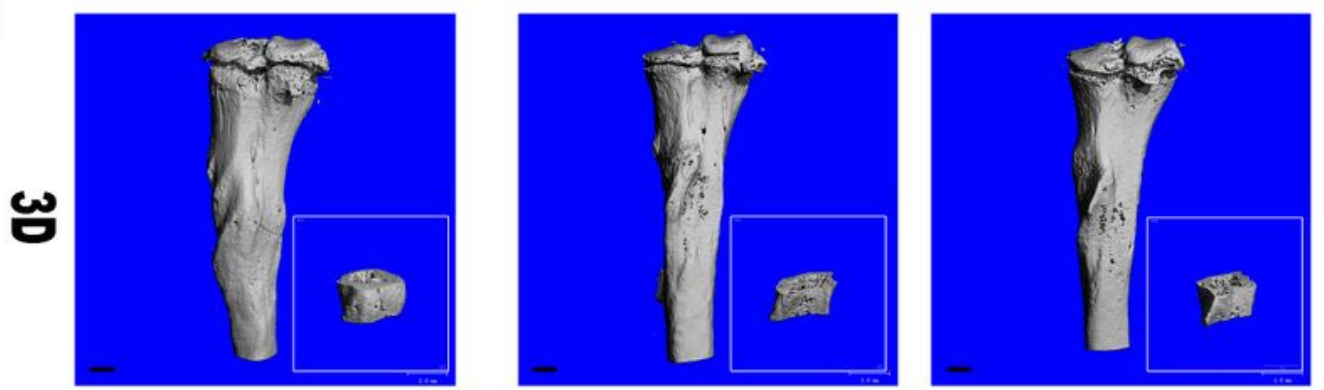

D

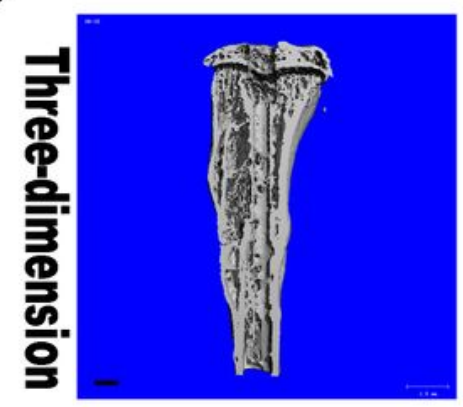

$\mathbf{E}$

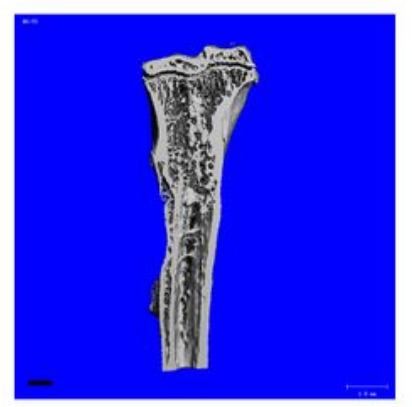

GFP-TNFa

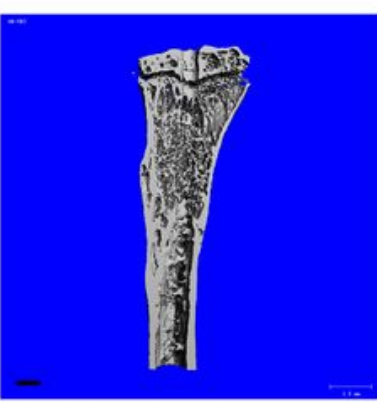

B7OE-TNFa
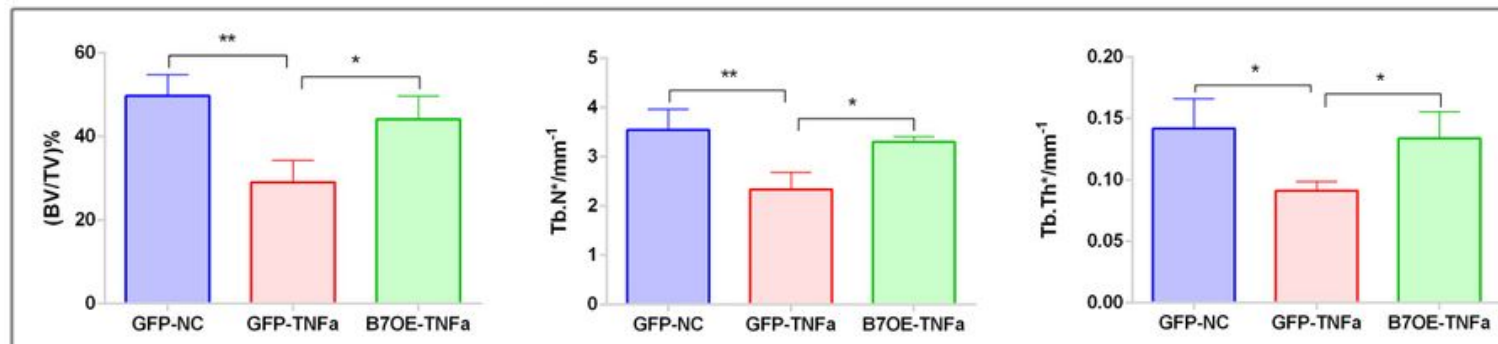

\section{Figure 6}

GFP-B7OE lentiviral particles protected fracture healing impaired by TNF- $a$ in a murine tibial fracture model. A-B) TNF-a $(100 \mathrm{ng} / \mathrm{mL})$ resulted in impaired fracture healing, which was rescued by the addition of GFP-B7OE lentiviral particles. The fracture healing effect was assessed by staining with the Masson's trichrome stain, Safranin 0 and fast green stain. Scale bars, 500 um. C-E) Microcomputed tomography analysis for bone healing. Scale bars, 500 um. GFP-NC: GFP expressed lentiviral particles (Negative 
Control); GFP-TNF-a: GFP expressed lentiviral particles with TNF-a; B7OE-TNF-a: HSPB7 overexpressed lentiviral particles with TNF-a. Data are expressed as mean \pm SD. Sample sizes used at minimum 6 animals per experimental procedure. ${ }^{*}, \mathrm{P}<0.05, * \star, P<0.01$, $* \star *, P<0.001$ compared with the control group.

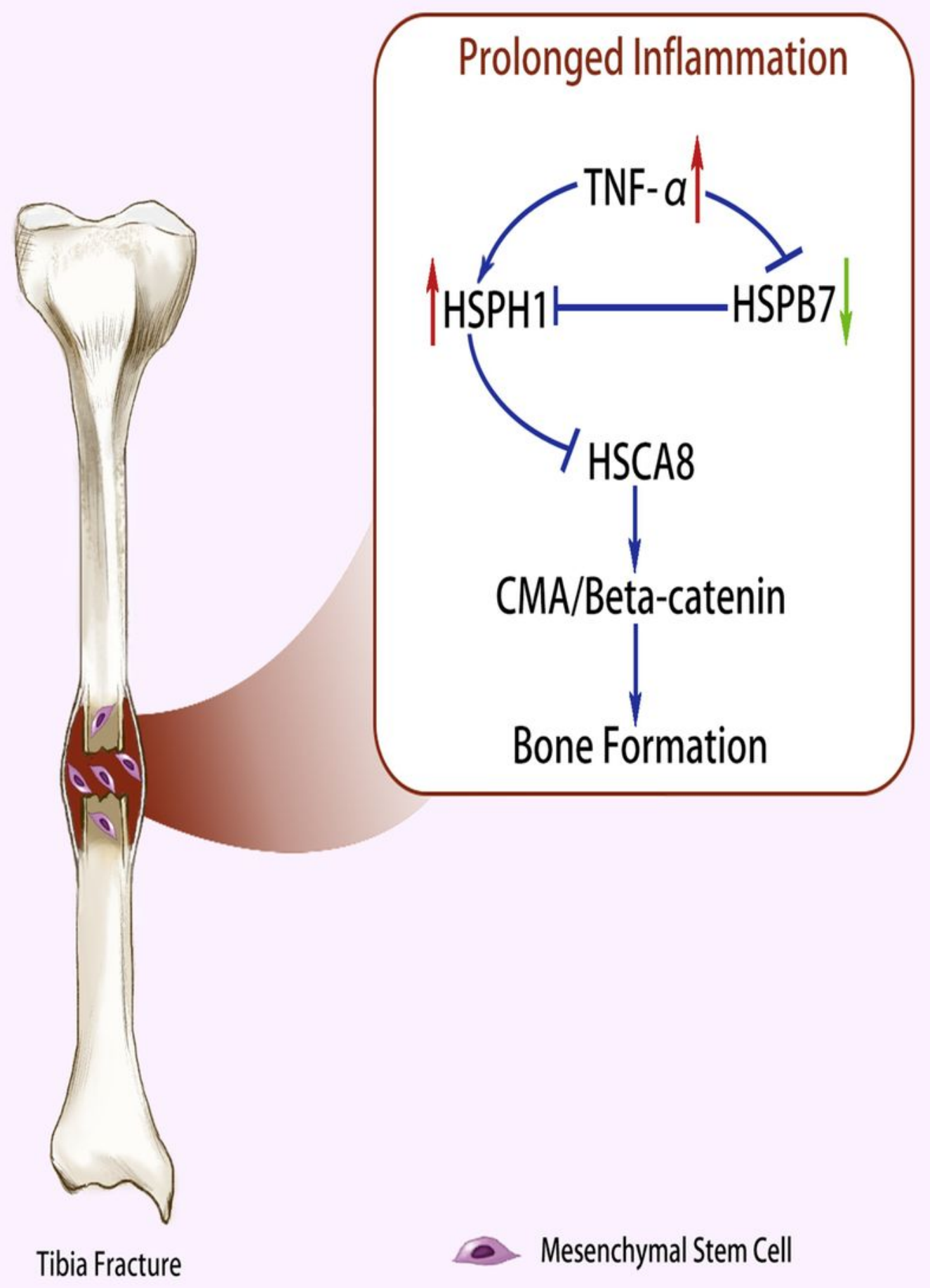

Figure 7

A heat shock protein family network including HSPB7, HSPH1 and HSCA8 protects the impaired osteogenesis induced by TNF-a via the CMA/ $\beta$-catenin pathway. 


\section{Supplementary Files}

This is a list of supplementary files associated with this preprint. Click to download.

- FigS1.jpg

- FigS2.jpg

- FigS3.jpg 